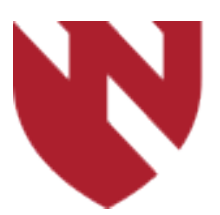

December 2019

\title{
Orthopaedic In-Training Examination (OITE) Preparation and Study Habits of Orthopaedic Residents: Revisited
}

\author{
Christopher F. Deans \\ University of Nebraska Medical Center \\ Emmett J. Gannon \\ University of Nebraska Medical Center \\ Joseph Morgan \\ University of Nebraska Medical Center \\ Elizabeth Lyden \\ University of Nebraska Medical Center \\ Matthew Mormino \\ University of Nebraska Medical Center
}

Tell us how you used this information in this short survey.

Follow this and additional works at: https://digitalcommons.unmc.edu/gmerj

Part of the Higher Education Commons, and the Medicine and Health Sciences Commons

\section{Recommended Citation}

Deans, C. F., Gannon, E. J., Morgan, J., Lyden, E., , Mormino, M. Orthopaedic In-Training Examination (OITE) Preparation and Study Habits of Orthopaedic Residents: Revisited. Graduate Medical Education Research Journal. 2019 Dec 13; 1(1).

https://digitalcommons.unmc.edu/gmerj/vol1/iss1/1

This Original Report is brought to you for free and open access by DigitalCommons@UNMC. It has been accepted for inclusion in Graduate Medical Education Research Journal by an authorized editor of DigitalCommons@UNMC. For more information, please contact digitalcommons@unmc.edu. 


\title{
Orthopaedic In-Training Examination (OITE) Preparation and Study Habits of Orthopaedic Residents: Revisited
}

\begin{abstract}
Introduction: The Orthopaedic In-Training Examination (OITE) is well-established as the cornerstone for educational evaluation of orthopaedic surgery residents. Great significance has been placed on the OITE, particularly as it has been found to correlate closely with successful completion of the American Board of Orthopaedic Surgery Part I Exam (ABOS I). Our study correlated different aspects of OITE study preparation, including resources and habits, with OITE performance.

Methods: An online survey was created to assess these different aspects and distributed to 163 programs across the United States for distribution to orthopedic residents in each program.

Results: Data analysis showed a positive correlation between OITE ranking and greater total hours devoted to studying $(r=0.26, p=0.0003)$, earlier start time for exam preparation $(r=0.25, p=0.0005)$, orthopaedic journal review (including Journal of Bone and Joint Surgery[r $=0.17, p=0.02]$ and American Academy of Orthopaedic Surgeons $[r=0.15, p=0.0475])$, review of prior OITE examinations $(r=0.20, p=$ $0.0054)$, and use of Orthobullets $(r=0.31, p<0.0001) .58 \%$ of respondents changed their study habits significantly over the course of residency. Most respondents stated they were able to study most effectively on primarily outpatient rotations, as well as pediatrics, sports, and hand orthopaedic rotations.

Conclusion: The results of this study may assist residents and residency directors to develop their curriculum and individual study plans to ensure success on the OITE, ABOS I, and, ultimately, their careers as orthopaedic surgeons.
\end{abstract}

\section{Keywords}

graduate medical education, residency education, orthopaedic education, orthopedic in-training examination

\section{Creative Commons License}

\section{c) (i) ()}

This work is licensed under a Creative Commons Attribution-Noncommercial-No Derivative Works 4.0 License. 


\section{Orthopaedic In-Training Examination (OITE) Preparation and Study Habits of Orthopaedic Residents: Revisited}

Christopher F. Deans¹, Emmett J. Gannon¹, Elizabeth Lyden², Joseph Morgan¹, Matthew Mormino

${ }^{1}$ University of Nebraska Medical Center, Department of Orthopaedics

${ }^{2}$ University of Nebraska Medical Center, College of Public Health

https://doi.org/10.32873/unmc.dc.gmerj.1.1.001

\begin{abstract}
Introduction: The Orthopaedic In-Training Examination (OITE) is well-established as an important metric for educational evaluation of orthopaedic surgery residents. Great significance has been placed on the OITE, particularly as it has been found to correlate closely with successful completion of the American Board of Orthopaedic Surgery Part I Exam (ABOS I). Our study correlated different aspects of OITE study preparation, including resources and habits, with OITE performance.
\end{abstract}

Methods: An online survey was created to assess resources utilized, time, and habits, and distributed to 163 programs across the United States for circulation to orthopaedic residents in each program.

Results: Data analysis showed a positive correlation between OITE ranking and greater total hours devoted to studying $(\mathrm{r}=$ $0.26, p=0.0003)$, earlier start time for exam preparation $(\mathrm{r}=0.25, \mathrm{p}=0.0005)$, orthopaedic journal review (including Journal of Bone and Joint Surgery $[\mathrm{r}=0.17, \mathrm{p}=0.02]$ and American Academy of Orthopaedic Surgeons $[\mathrm{r}=0.15, \mathrm{p}=0.0475])$, review of prior OITE examinations $(r=0.20, p=0.0054)$, and use of Orthobullets $(r=0.31, p<0.0001)$. Fiftyeight percent of respondents changed their study habits significantly over the course of residency. Most respondents stated they were able to study most effectively on primarily outpatient rotations, as well as pediatrics, sports, and hand orthopaedic rotations.

Conclusion: The results of this study may assist residents and residency directors to develop their curriculum and individual study plans to ensure success on the OITE, ABOS I, and, ultimately, their careers as orthopaedic surgeons.

\section{Introduction}

The Orthopaedic In-Training Examination (OITE) was established in 1963 by the American Academy of Orthopaedic Surgeons with the intention to help orthopaedic surgery residents and residency programs evaluate the effectiveness of their educational curriculum. ${ }^{1}$ The OITE is well-established as the cornerstone for educational evaluation of orthopaedic surgery residents in the United States. ${ }^{1,2,3}$ According to Evaniew et al., program directors in the United States rated OITE scores as 'Very Important' or 'Extremely Important', and also endorsed greater consequences for those who scored poorly ${ }^{4}$. The greater significance placed on the OITE in the U.S. is well-validated, having been repeatedly shown that OITE results correlate closely with successful completion of the American Board of Orthopaedic Surgery (ABOS) Part I Exam. 5,6,7,8

Some have sought to evaluate different aspects of orthopaedic residents' preparation for the OITE. Miyamoto et al. surveyed 44 residents in their program and found a statistically significant correlation between successful OITE performance and frequent review of current orthopaedic journals, daily orthopaedic reading, and greater number of hours committed to studying. ${ }^{9}$ Evaniew surveyed 331 residents and found that in preparation for the OITE most residents strongly favored the use of prior OITE exams as well as the American Academy of Orthopaedic Surgeons (AAOS) self-assessment questions, the 'AAOS Comprehensive Orthopaedic Review' textbook, and the Journal of the Academy of Orthopaedic Surgeons. ${ }^{4}$ They also found an 'extreme' level of importance assigned to web-based resources, although those specific resources were not named or evaluated. In a separate study, Laporte et al surveyed 360 residents and found a positive correlation between OITE score and use of the Journal of American Academy of Orthopaedic Surgeons, but no correlation with the use of traditional standard reference textbook for studying. ${ }^{10}$ While this is only a small sample of past studies evaluating OITE preparation resources, they demonstrate the common findings noted in the literature.

With the expansion of orthopaedic education materials and resources, in particular the rapid growth of internet-based educational resources and curricula, we find it worthwhile to re-visit the topic. We also believe that with the effort to survey the residents across the entire U.S., a broader representative sample of data can be obtained. We therefore sought in the present study to evaluate the: (1) most popular study resources utilized by residents to study for OITE; (2) amount of time devoted to each study resource; (3) amount of dedicated time available for study; (4) correlation between these various factors to OITE performance.

\section{Methods}

Study Design: Program coordinators or administrative assistants at 163 United States orthopaedic surgery residency programs accredited by the ACGME were contacted via e-mail with a letter of explanation and a blinded Web-based survey (Supplemental Figure 1). The program coordinator and administrative assistants were asked to distribute the letter and web link to their residents for their voluntary participation in the survey. Survey response data were collected for a total of 8 weeks, with a goal response rate of $10 \%$ of U.S. orthopaedic residents, in line with past physician survey response rates. ${ }^{11,12,13}$ Full confidentiality of survey responses was preserved by the double-blinded properties within the Webbased survey service. There were no exclusion criteria. Inclusion criteria included any orthopaedic surgery resident at ACGMEaccredited U.S. orthopaedic surgery residency programs who had completed the OITE the same year as the survey was distributed. This study was determined to be Institutional Review Board (IRB) exempt.

Survey Development: This survey was adapted from a similar survey used by Miyamoto et al. (Supplemental Figure 2), to which questions were added to gather data on more recent educational resources. In order to optimize the clarity and response rate of the survey, it was first piloted to residents at the University of Nebraska Medical Center. Feedback helped the researchers further improve ease of response, clarify any ambiguity of questions or terms, and minimize responders' time commitment. A combination of 7-point Likert scales, multiple-choice questions, multiple-answer questions, "yes 
and no" questions, and free response were used (Supplemental Figure 1). A cover letter was included on the introductory page of the survey administered via SurveyMonkey.com. Completing the survey served as consent to participate.

Statistical Analysis: Descriptive statistics counts and percentages were used to summarize the data, with analysis by an experienced biostatician. Spearman correlation coefficients were used to assess the association between OITE rank and specific habits during residents' training, including exam prep start time, total hours studying, specific study techniques, and USMLE Step 1 and 2 scores. Associations of study habits (with yes/no responses) and rank percentile (collapsed into $\geq 90 \%, 89-75 \%, 74-50 \%$ and 49-1\%) were assessed with Fisher's exact test.

\section{Results}

One hundred ninety-four residents responded to the survey out of 3,500 residents, which translated to a response rate of approximately 5\%. Respondents were roughly divided into fifths between PGY-1 and PGY-5 (19.6\%, 20.1\%, 21.1\%, 21.6\%, and 17.5\%, respectively) and reported OITE percentile rank following a representative distribution with most scores between the 25th and 90th percentiles (Figures 1 and 2).

Higher OITE rank percentiles were associated with greater total hours devoted to studying $(r=0.26, p=0.0003)$ and earlier start for exam preparation $(\mathrm{r}=0.25, \mathrm{p}=0.0005)$ (Table 1). Sixty-six percent of respondents began preparing 2 or more months prior to examination, with approximately $60 \%$ devoting greater than 40 hours for preparation time. OITE ranking was positively associated with greater USMLE Step 1 score $(r=0.31$, $\mathrm{p}<0.0001)$ and USMLE Step 2 score $(\mathrm{r}=$ $0.36150, \mathrm{p}<0.0001)$ taken during medical school (Table 1).

A weak correlation was identified between higher OITE rank percentile and reading Journal of Bone and Joint Surgery $(\mathrm{r}=0.17$, $\mathrm{p}=0.02$ ) as well as Journal of the American Academy of Orthopaedic Surgeons $(\mathrm{r}=0.15$, $\mathrm{p}=0.0475$ ). A weak correlation was found between higher rank percentile for those that utilized reviewing prior OITE examinations $(\mathrm{r}=0.20, \mathrm{p}=0.0054)$ and moderate correlation with use of Orthobullets.com ( $\mathrm{r}=$ $0.31, \mathrm{p}<0.0001)$.

No correlation was found between OITE performance and use of review textbooks $(r=0.06, p=0.43)$, self-assessment

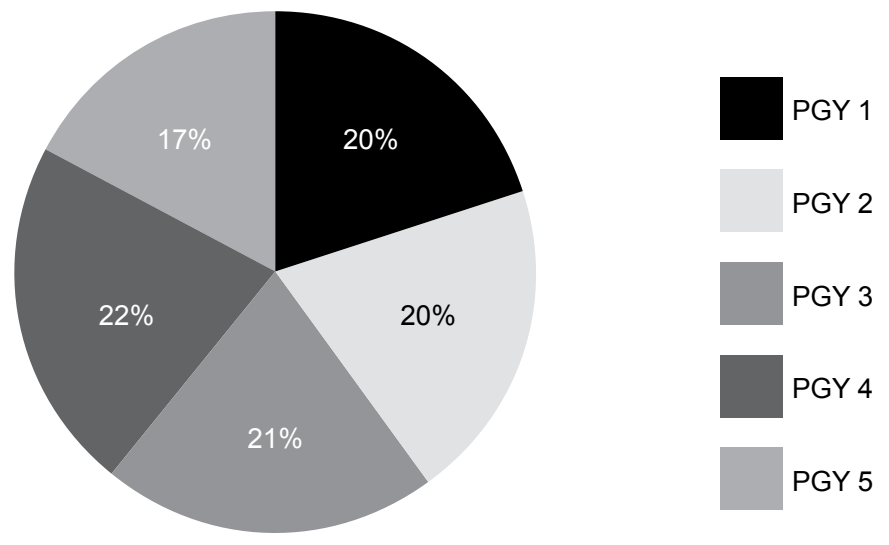

Figure 1. Survey response percentile by post-graduate year (PGY) demonstrating good distribution between each year of residency.

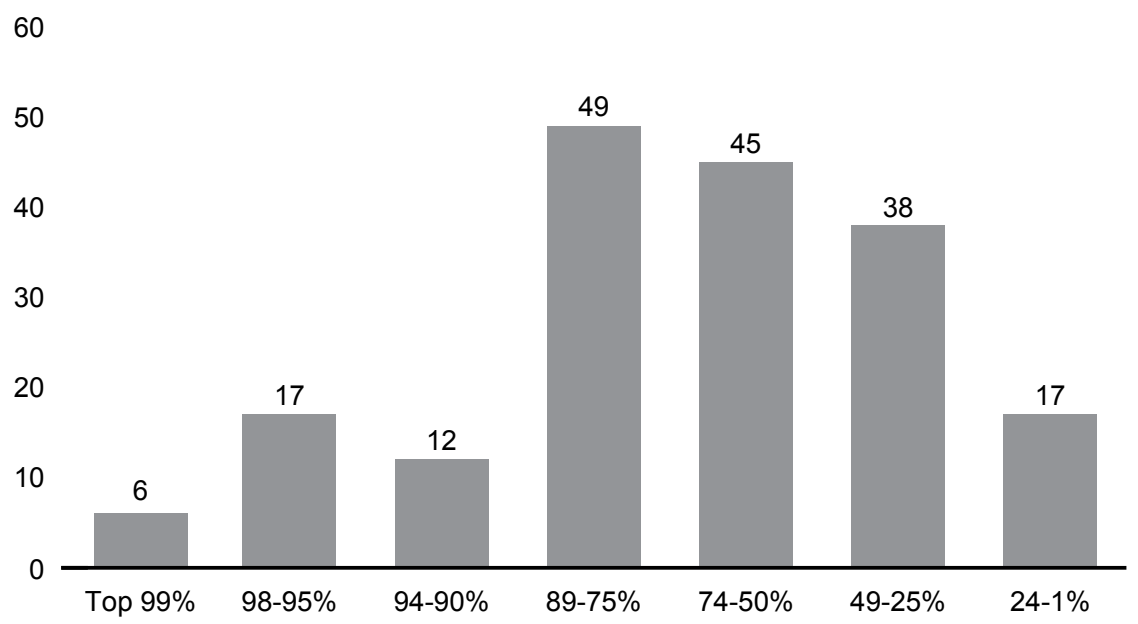

Figure 2. Number of survey responders reporting each OITE percentile rank demonstrating a distribution grossly similar to expected national rank distributions.

\section{Table 1.}

Factors, including study materials and techniques, assessed in this study and their association with OITE percentile rank.

\begin{tabular}{|c|c|}
\hline $\begin{array}{l}\text { Factors Associated with Higher } \\
\text { Percentile Rank (R-value) }\end{array}$ & $\begin{array}{l}\text { Factors Not Associated with Higher } \\
\text { Percentile Rank (R-value) }\end{array}$ \\
\hline Greater total hours devoted to study $(0.26)^{\star}$ & Review of orthopaedic textbook (0.06) \\
\hline Earlier start time for study $(0.25)^{\star}$ & Use of self-assessment examination (0.08) \\
\hline Greater USMLE Step 1 and Step 2 Score $(0.31 ; 0.36)^{*}$ & Review of lecture notes (0.03) \\
\hline Reading Journal of Bone and Joint Surgery $(0.17)^{*}$ & Giving presentations for conferences $(-0.09)$ \\
\hline $\begin{array}{l}\text { Reading Journal of American Academy } \\
\text { of Orthopaedic Surgeons }(0.15)^{\star}\end{array}$ & Skills labs / cadaver dissections (-0.09) \\
\hline Reviewing prior OITE examinations $(0.21)^{*}$ & Use of flashcards $(-0.02)$ \\
\hline Use of Orthobullets.com $(0.31)^{*}$ & Miller's Review Course (-0.01) \\
\hline
\end{tabular}


examinations $(\mathrm{r}=0.08, \mathrm{p}=0.27)$, flashcards $(\mathrm{r}=-0.02, \mathrm{p}=0.74)$, review of lecture notes $(\mathrm{r}=0.04, \mathrm{p}=0.62)$, giving presentations for conferences $(r=-0.09, p=0.22)$, skills laboratories/cadaver dissections $(\mathrm{r}=-0.1, \mathrm{p}=$ $0.19)$ or Miller's Review Course ( $r=-0.01$, $\mathrm{p}=0.88)$ (Table 1).

Study habits were reported by respondents in 'yes,' 'no,' or ' $n / a$ ' format. Of the $91 \%$ of respondents that attended didactic lectures and conferences, $26 \%$ responded that they do take notes with only $17 \%$ then reviewing those notes in the future. Thirty-seven percent stated they have a personal structured reading schedule. Participation in reading orthopaedic journals was quite low, with Journal of Bone and Joint Surgery and Journal of the American Academy of Orthopaedic Surgeons being read monthly at $18 \%$ and $29 \%$, respectively. These journals were also considered 'not helpful' or 'minimally helpful' by $84 \%$ of those who used this as a study resource. In contrast, greater than $98 \%$ of respondents not only use Orthobullets but said it was more than moderately helpful. Fifty-three percent of respondents changed their study techniques or resources based on feedback from prior OITE exams.

The rotations most conducive for study were noted to be primarily outpatient rotations $(29 \%)$, pediatrics $(15 \%)$, sports $(12 \%)$, and hand/upper extremity (10\%) (Figure 3 ). Rotations least conducive for studying for OITE included trauma (62\%), joint replacement $(11 \%)$, tumor/musculoskeletal oncology (8\%) (Figure 4).

\section{Discussion}

The OITE represents one of many tools used to assess resident knowledge during orthopaedic training. It has been demonstrated that better OITE results correlate with success on the ABOS Part I Examination. 5,6,7,8 For these reasons, both program directors and residents alike may be interested in determining what study habits and resources most support OITE success. Miyamoto et al. set the precedent in regard to the subject in 2007, with their study on OITE preparation amongst residents. ${ }^{9}$ Our study poses the same question on a broader stage with a larger sample of programs and residents from the entire U.S.

Our results further emphasize a foundational value for success in residency and lifelong practice - time devoted to study and preparation is essential. There was a strong correlation with earlier study start date and greater total hours devoted to studying with

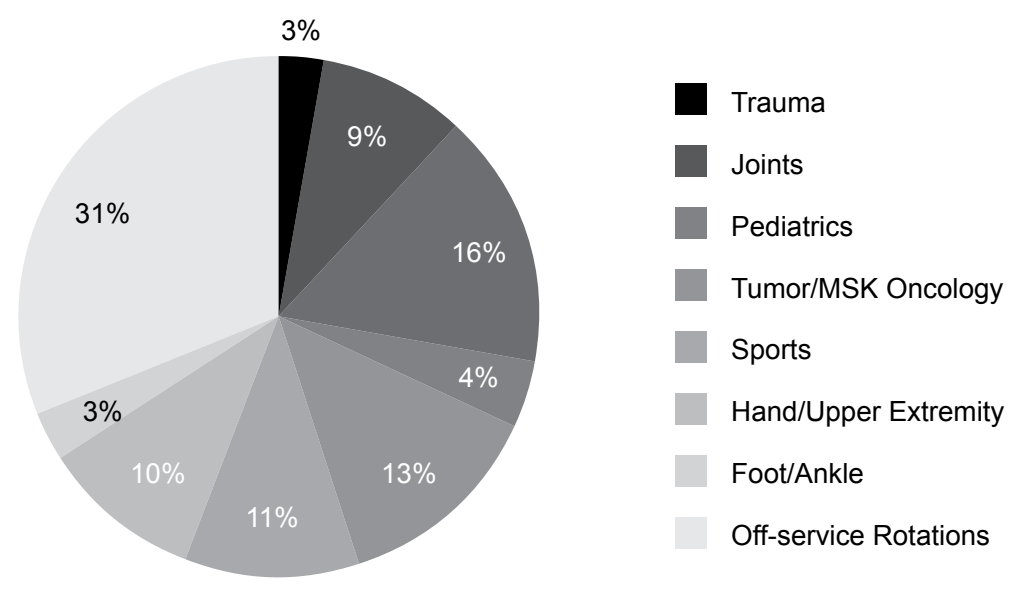

Figure 3. Respondent reports of rotations on which one is able to most effectively study.
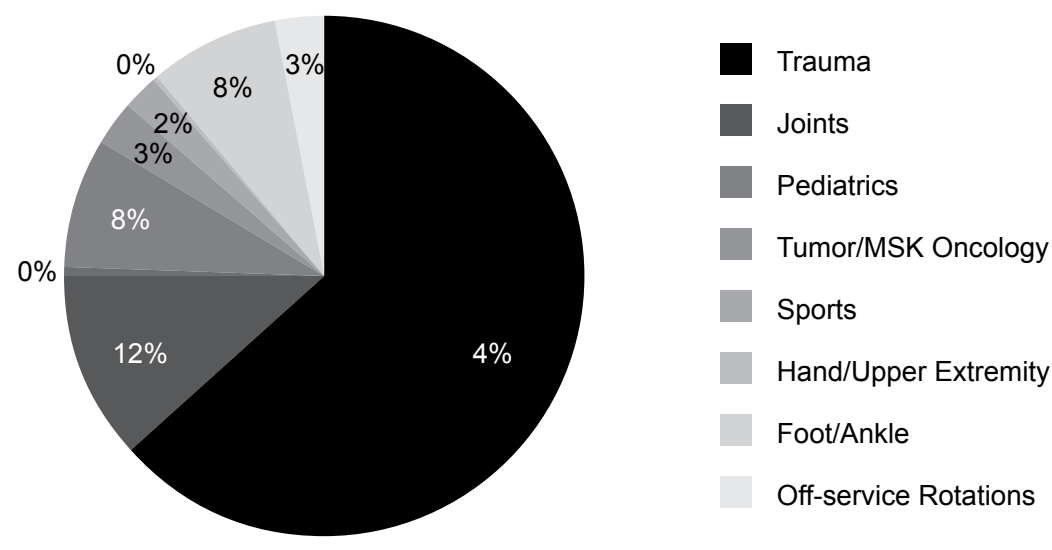

Figure 4. Respondent reports of rotations on which one is least able to study effectively.

higher OITE percentile rank. The results of our study suggest that 60 hours of preparation may be a discriminator as noted by the sharp contrast in score distribution above and below that mark. Above 60 hours there appears to be a plateau in improvement of score until one reaches 80 or greater hours. This concept of a peak or plateau in test results after preparation for professional examinations has been established in USMLE Step 1 preparation literature, which correlates with our own findings. ${ }^{14,15}$

The importance of sufficient time devoted to preparation should not be under-emphasized. Respondents reported their ability to study more effectively during what are considered less time-intensive services, such as outpatient rotations including off-service rotations, as well as pediatrics, sports, and hand and upper extremity orthopaedic rotations. However, this remains a subjective report on how residents believed they studied while on those rotations. While our study did not assess the value of clinical education on OITE performance, we would expect that clinical education remains an important source of knowledge that would contribute to performance on the OITE.

Our results indicate that there has been a shift in what residents with good OITE scores consider to be the most effective and efficient resources. According to Miyamoto et al., use of Journal of Bone and Joint Surgery (JBJS) and Journal of the American Academy of Orthopaedic Surgeons (JAAOS) as study resources demonstrated strong correlations with OITE success in 2007. ${ }^{9}$ The majority of respondents in our study reported that they rarely read JBJS or JAAOS and that they either did not use these journals, or found them minimally helpful in OITE preparation. In contrast, a stronger correlation with OITE success was found with the online resource Orthobullets.com. The reasons for this shift are likely multifactorial. First, in the past 10 years a new generation of learners who have grown up using more computer or online-based learning platforms throughout their education have entered residency. ${ }^{16,17,18}$ 
It is likely that Orthobullets is a familiar platform and has been effective for them in the past. ${ }^{19}$ Second, Orthobullets.com has become ubiquitous with orthopaedic resident education due to its ease of use, bulleted format for quick review, and ability to be "at your fingertips" at all times. ${ }^{20,21}$ Lastly, as journals are released only monthly they do not provide for sustained study material; rather, they provide an excellent update on basic and clinical research in musculoskeletal science, as well as review articles. In contrast, Orthobullets.com allows for individual topic review with primary literature citation with the advantage of various study plans available for structured topic review. ${ }^{20,21}$ Additionally, Orthobullets.com represents a new resource that was not available at the time of the work published by Miyamoto et al., broadening the landscape of resources accessible to residents today.

Interestingly, respondents also indicated that they are much more likely to prepare for the OITE by studying prior OITE exams than source material. The vast majority of respondents rated studying prior examinations as "helpful" or "extremely helpful", and there was a stronger positive correlation with prior OITE exam review and higher OITE percentile rank than was seen with journal review. This is a strong indicator of the way that residents view the OITE as it has evolved over the years. It is quite likely that residents are most interested in preparing for the sake of achieving a greater score than approaching

\section{References}

1 Mankin, H. The orthopaedic in-training examination (OITE). Clin orthop relat res. Mar 1971; (75), 108-16.

2 Osbahr, D. Orthopaedic in-training examination: an analysis of the sports medicine section. Amer $J$ of Sports Med. Dec 2010

3 Taylor, B. Analysis of the trauma section of the orthopaedic in-training examination. Orthopedics. Jul 2011; 34(7), e261-66. Retrieved from Health.com/ Orthopaedics: Healio.com /Orthopedics/20120525-28

4 Evaniew, N. The orthopaedic in-training examination: perspectives of program directors and residents from the United States and Canada. J Surg Educ. Jul 2013; 70(4), 528-536.

5 Klein, G. Passing the boards: can USMLE and Orthopaedic In-Training Examination scores predict passage of the ABOS Part-I Examination? J Bone Joint Surg Am. 2004; 86(5), 1092-1095.

6 Crawford, C. Relationship among United States Medical Licensing Step I, orthopedic in-training, subjective clinical performance evaluations, and American board of orthopaedic surgery examination scores: a 12-years review of an orthopaedic surgery residency program. J Surg Educ. Mar 2010; 67(2), 71-78.

7 Dyrstad, B. Predictive measures of a resident's performance on written orthopaedic board scores. Iowa Orthop J. 2011; (31), 238-243.

8 Swanson, D. Utility of AAOS OITE scores in predicting ABOS Part I outcomes: AAOS exhibit selection. J Bone Joint Surg Am. 2013; (95), e84. preparation as a means of accumulating greater command and understanding of subject matter. This is a common finding in modern medical training, and likely no different than preparation for USMLE Step 1 and Step $2 .{ }^{22,23}$ It is worth noting that, although the data indicate that residents are moving away from journals and primary literature for OITE preparation, this does not necessarily mean that residents are avoiding them outside of the OITE preparation period.

Another foundational value for successful learning was reinforced by our results: be dedicated to intent, but flexible in approach. Greater than half of our respondents said that their study habits changed during residency, which is indicative that most residents are sensitive to feedback obtained in their score report. While our study does not describe residents' approach to improving their overall understanding, one might expect that residents would be flexible in their approach to most effectively and efficiently develop their knowledge base and skills as orthopaedic surgeons outside of the exam, as well.

The relatively small sample size and response rate do represent limitations to this study. The sample size obtained in this study was below our goal of a $10 \%$ response rate. It is well-known that low response rates are a commonality amongst survey-centered studies, particularly attempting to collect from such a large number of potential participants. ${ }^{11,12,13}$ Although the response rate in this study is less than desired a good distribution of responses between PGY-1 and PGY-5 (with each PGY representing approximately $20 \%$ of the overall sample), as well as between different OITE percentile rank was noted (roughly representing a Bell curve). Also, similar to all retrospective survey studies, recall bias is possible, which limits the objectivity of our data. Our survey was conducted within 3 months of OITE score release in an effort to minimize this bias, but it cannot be eliminated entirely.

As is so aptly described by Mendezabal, performance on an exam is the result of a combination of ability with study habits and attitudes. ${ }^{24}$ Most orthopaedic residents can be assumed to have achieved a certain level of aptitude in ability, as indicated by their acceptance into orthopaedic residency. The correct study habits, however, can be a critical factor in performance on the OITE. We understand with the ever-greater significance placed on OITE performance and its positive correlation with success of licensing examinations that determining the study habits can be of high importance. We believe that the information in this and other studies on the subject may assist orthopaedic residents and residency program directors to develop their curriculum and individual study plans with the most effective and efficient resources to ensure their success on the OITE and, ultimately, their careers as orthopaedic surgeons.
9 Miyamoto, R. Orthopaedic surgery residents' study habits and performance on the orthopaedic in-training examination. Am J Orthop. 2013; (36), e185-e188.

10 Laporte et al. Educational resources for the orthopaedic in-training examination. J Surg Educ. 2010; 67(3), 135-138.

11 Cull, W. Response rates and response bias for 50 surveys of pediatricians. Health services research. 2005; (40), 213-226.

12 Yarger, J. Characteristics in response rates for surveys administered to surgery residents. $J$ Surg. 2013; 154(1), 38-45.

13 Cummings, S. Reported response rates to mailed physician questionnaires. Health services research. $2001 ; 35(6), 1347-1355$.

14 Dallaghan, B. Techniques in preparation for Step 1 of the United States Medical Licensing Examinations. 2014

15 Black, K. Orthopaedic in-training examination scores: a correlation with USMLE results. J Bone Joint Surg Am. 2006; 88(3), 671-676.

16 Nicholas, A. Preferred learning methods of the millenial generation. Faculty and Staff - Articles \& Papers. 2008.

17 MacKenzie, J. The world wide web: redefining medical education. J Amer Med Assoc. 1997; 278(21), 1785-86.

18 Ruiz, J. The impact of e-learning in medical education. Acad Med. 2006; 81(3), 207-12.
19 Cook, D. Internet-based learning in the health professions. J Amer Med Assoc. Sept 2008; 300(10), 1181-96.

20 Taylor, B. Achieving educational excellence: a strategic initiative to enhance orthopedic resident academic performance. J surg ed. Jun 2011; 68(3), 162-166.

21 Boody, B. Validation of a web-based curriculum for resident education in orthopedic surgery. J surg educ. Nov 2016; 73(6), 1060-65.

22 Tompkins, J. Money for nothing? The problem of the board-exam coaching industry. $N$ Eng J Med. Jul 2011; 365(2), 104-5.

23 Culler, R. et al. Test anxiety and academic performance: The effects of study-related behaviors. J of Educ Psych. 1995; 72(1), 16-20.

24 Mendezabal et al. Study habits and attitude: the road to academic success. Feb 2013. Retrieved 3 2017, from Open access repository education. 